\title{
Nondestructive Detection of the Gel State of Preserved Eggs Based on Dielectric Impedance
}

\author{
Cheng-Han Li ${ }^{1}$, Chun-Hung Hsieh ${ }^{2}$, Cheng-Chu Hung ${ }^{1}$ and Ching-Wei Cheng ${ }^{3, *}$ \\ 1 Department of Bio-Industrial Mechatronics Engineering, National Chung Hsing University, \\ Taichung 402, Taiwan; cghan@smail.nchu.edu.com.tw (C.-H.L.); ackt223@gmail.com (C.-C.H.) \\ 2 Department of Information Management, National Taichung University of Science and Technology, \\ Taichung 404, Taiwan; guu@nutc.edu.tw \\ 3 College of Intelligence, National Taichung University of Science and Technology, Taichung 404, Taiwan \\ * Correspondence: cwcheng@nutc.edu.tw; Tel.: +886-4-2219-5795
}

Citation: Li, C.-H.; Hsieh, C.-H.; Hung, C.-C.; Cheng, C.-W.

Nondestructive Detection of the Gel State of Preserved Eggs Based on Dielectric Impedance. Foods 2021, 10, 394. https://doi.org/10.3390/foods 10020394

Academic Editors: Françoise Nau and Valerie Lechevalier

Received: 23 December 2020

Accepted: 7 February 2021

Published: 11 February 2021

Publisher's Note: MDPI stays neutral with regard to jurisdictional claims in published maps and institutional affiliations.

Copyright: (c) 2021 by the authors. Licensee MDPI, Basel, Switzerland. This article is an open access article distributed under the terms and conditions of the Creative Commons Attribution (CC BY) license (https:// creativecommons.org/licenses/by/ $4.0 /)$.

\begin{abstract}
After completing the production of preserved eggs, traditionally, the degree of gelling is judged by allowing workers to tap the preserved eggs with their fingers and sense the resulting oscillations. The amount of oscillation is used for the quality classification. This traditional method produces varying results owing to the differences in the sensitivity of the individual workers, who are not objective. In this study, dielectric detection technology was used to classify the preserved eggs nondestructively. The impedance in the frequency range of $2-300 \mathrm{kHz}$ was resolved into resistance and reactance, and was plotted on a Nyquist diagram. Next, the diagram curve was fitted in order to obtain the equivalent circuit, and the difference in the compositions of the equivalent circuits corresponding to gelled and non-gelled preserved eggs was analyzed. A preserved egg can be considered an RLC series circuit, and its decay rate is consistent with the decay rate given by mechanical vibration theory. The Nyquist diagrams for the resistance and reactance of preserved eggs clearly showed that the resistance and reactance of gelled and non-gelled eggs were quite different, and the classification of the eggs was performed using Bayesian network (BN). The results showed that a BN classifier with two variables, i.e., resistance and reactance, can be used to classify preserved eggs as gelled or non-gelled, with an accuracy of $81.0 \%$ and a kappa value of 0.62 . Thus, a BN classifier based on resistance and reactance demonstrates the ability to classify the quality of preserved egg gel. This research provides a nondestructive method for the inspection of the quality of preserved egg gel, and provides a theoretical basis for the development of an automated preserved egg inspection system that can be used as the scientific basis for the determination of the quality of preserved eggs.
\end{abstract}

Keywords: preserved egg; nondestructive; dielectric method; impedance

\section{Introduction}

Preserved eggs are mainly produced by marinating fresh eggs. Marinated duck eggs are much easier to store than fresh duck eggs, and they contain large amounts of protein and amino acids [1]. Preserved eggs exhibit a unique smell and taste that can enhance the appetite, and they are well known in Asia as a traditional Chinese food. Famous preserved egg dishes-such as tofu with preserved egg, three cups of preserved egg, and lean porridge with preserved egg-are very popular. Preserved eggs are also exported to other countries, such as the United States, Canada, and Japan, where their unique aroma is appreciated. According to statistics from the Taiwan government and the Duck Association of the Republic of China, Taiwan's annual output of duck eggs is approximately 460 million, and the yearly total value is approximately US \$56 million. The majority of the duck eggs are converted into processed duck eggs. The preservation of the eggs depends on pickling using alkaline substances. This causes chemical reactions involving the proteins inside the 
eggs, and results in their solidification. In general, the methods of producing preserved eggs can be divided into three categories:

- Coating method: The fresh eggs are wrapped in a strong alkaline mud consisting mainly of a mixture of plant ash, white lime, and rice husks, and then they are kept at room temperature for 30-40 days [2].

- Immersion method: This is currently the primary method of making preserved eggs. Salt, tea, and other substances are mixed with sodium hydroxide or potassium hydroxide in particular proportions. In order to adjust the osmotic pressure of the alkaline solution in the egg, and to improve the success rate of the process, minute amounts of copper, zinc, iron, and metal salt compounds are added to the pickling liquid. Using this method, the time taken to produce the preserved eggs depends on the concentration of the alkaline solution, the type and concentration of metal compounds used, and the immersion temperature. Generally, in this way, preserved eggs can be produced in a shorter time than using traditional methods that work at room temperature [3,4].

- Combined method: This method involves a combination of the coating and immersion methods. The fresh eggs are wrapped in paper and put into a plastic bag, and a small amount of alkaline solution is added. The paper with the alkaline solution indirectly immerses the egg in the alkaline liquid, and then the egg is left at room temperature for 30-40 days to mature [2].

Because of the interaction between the duck egg and these alkaline substances, the duck egg becomes a preserved egg. The shell of a standard preserved egg is easy to remove, and the appearance of the preserved egg's albumen is smooth, and similar to that of tan jelly. Patterns shaped like pine needles or needle-like crystals are formed as the egg yolk moves to the egg albumen. The egg yolk becomes solid, and typically appears dark green or grass green. If the gel of the preserved egg is incomplete, a thick, brown liquid may flow out when the eggshell is removed; the eggshell may also stick to the surface of the albumen and be difficult to remove. Such eggs also emit a pungent smell, which may cause nausea. The mature preserved eggs are removed from the soaking bucket and dried. After drying, the quality of the preserved eggs is manually determined by tapping the eggs and sensing the amount of oscillation. Preserved eggs that are gelled to the normal standard oscillate strongly when they are tapped with a finger; this is the primary detection method that is currently used in preserved egg factories. The use of this method may cause injury to factory workers. Eventually, the workers can lose sensitivity in their fingers, which also leads to misclassification. No standard way of quantifying the degree of gelling of a preserved egg exists; therefore, long-term experience is needed to identify the degree of gelling using the tapping method.

The quality of many agricultural products is estimated by measuring their electrical characteristics. For example, one method involves inserting steel needles into fruit tissue that becomes an electrode; the voltage and frequency are then varied, and the change in the electrical impedance is measured. Thus, changes in the internal structure of different varieties of apples during storage can be estimated from the changes in the impedance at different frequencies [5]. The internal moisture content of the yolk and protein in poultry eggs changes with time. In order to determine their storage time [6], the moisture content can be determined by measuring the electrical conductivity using an alternating-current voltage, which is a destructive test. In the case of nondestructive methods, most of the dielectric parameters are measured using capacitive methods or open-circuit terminal probes. Ghaderi et al. (2018) used a nondestructive method employing parallel plate capacitors to measure the dielectric constant and loss factor by changing the voltage frequency to classify poultry eggs as fertilized, nonfertilized, or dead [7]. The nondestructive testing of eggs using parallel plate capacitors, changing the frequency range of the sine wave voltage within the range of $40 \mathrm{kHz}-20 \mathrm{MHz}$, and measuring the eggs' dielectric parameters can be used to predict the freshness of poultry eggs [8]. Other studies, in which dielectric properties were used to determine the quality of different products, involved fruits [9], agricultural products [10], and foods [11]. However, research on the detection of preserved 
egg gel is scarce. In one study, Chen et al. (2011) proposed the use of the plastic sticktapping method to determine the quality of gelling in preserved eggs from which the shell was not removed [12]. The preserved eggs were tapped using a constant force, and an accelerometer was used to determine the amount by which the eggs oscillated. The rate at which the oscillations decayed was low in the case of gelled eggs, and high if the eggs had not been gelled. The corresponding changes in the electrical impedance of the biological samples may vary according to the frequency of the external electrical field, with tissue structure and composition differences $[13,14]$. Therefore, the electrical parameters from fitting circuits can be conveniently explored by impedance, resistance, and reactance in order to describe the samples' response and determine the preserved eggs' state.

The purpose of this research was to investigate a nondestructive dielectric method to determine whether the gelling of preserved eggs is complete. The classification of eggs as either gelled or non-gelled preserved eggs was based on their dielectric impedance. This method could possibly replace the traditional method of tapping the preserved eggs with fingers to classify the gel quality. The difference between the results that were obtained based on the detection of the dielectric properties and those obtained using the tapping method [12] was also investigated. Equivalent circuits for completely and incompletelygelled preserved eggs were established, and dielectric impedance was used to classify the gelling quality of the preserved eggs.

\section{Materials and Methods}

\subsection{Samples}

The samples of gelled and non-gelled preserved eggs used in this study were produced by the Chuan-ming egg company based in Chiayi County, Taiwan. The preserved eggs were all produced from fresh duck eggs by immersing them in an alkaline solution. All of the preserved eggs were produced in the same production conditions following the same procedures. The gelled and non-gelled preserved eggs were produced by marinating a large number of preserved eggs. After the production of the preserved eggs was complete, the factory workers tapped them to determine their gel status. In this study, the eggs shown in Figure 1a,b were defined as completely-gelled preserved eggs. When these shells were removed, the albumen at the small end of the preserved eggs was elastic and smooth, and no thick liquid was present; the albumen had also completely solidified. Figure $1 \mathrm{~b}$ shows high-quality preserved eggs that exhibit 'pine flowers' in the egg whites. This type of preserved egg is called a 'pine flower preserved egg.' Figure 1c,d shows non-gelled preserved eggs that contained a thick liquid or liquid albumen at the small end after the shell was removed. In this study, the eggs shown in Figure 1c,d were defined as non-gelled preserved eggs, or incompletely-gelled preserved eggs.

In this study, we considered 176 samples of gelled and non-gelled preserved eggs. The impedance and loss factors of the preserved eggs were measured using an LCR-6300. After all of the samples were investigated, the eggs were first classified as gelled or non-gelled by Bayesian network (BN) classification in machine learning, and 30 gelled preserved eggs and 30 non-gelled preserved eggs were used for the training of the machine-learning model. In addition, 116 preserved eggs were used for the classification accuracy verification.

\subsection{Impedance Spectrum Measurements}

The measurements of the preserved eggs were made indoors at an average temperature of $25{ }^{\circ} \mathrm{C}$ and a relative humidity of $43 \%$. The impedance spectrum measurements were taken using an LCR-6300 (Good Will Instrument Co., Ltd., New Taipei City, Taiwan) with homemade parallel plate capacitors. The LCR-6300 is generally used to measure the resistance, inductance, and capacitance of electronic components, and its output and measurement frequency range are 10-300 kHz. The LCR-6300 was used to measure the impedance of the preserved eggs using parallel plate capacitors. Figure 2a shows the measurement setup. The parallel plate capacitor was comprised of two pieces of acrylic $(75 \times 55 \mathrm{~mm})$, two pieces of conducting-cloth-covered foam $(60 \times 55 \mathrm{~mm})(\mathrm{U}-\mathrm{TEK}$ EMI 
Corp., New Taipei City, Taiwan), and four springs. The conducting foam was compressible, which meant that the contact area and the tightness of the contact between the conducting plane and the preserved egg could be increased. The four springs in the parallel plate capacitor allowed the distance between the parallel plates to be automatically adjusted according to the size of the egg. The parallel plate capacitor is shown in Figure 2a. The measurements were made on individual eggs without removing the shell. Each pair of numbers (1-4) in Figure $2 b$ represents one pair of contact positions of the parallel plates; the data from the four different pairs of positions were averaged after the measurements were taken.

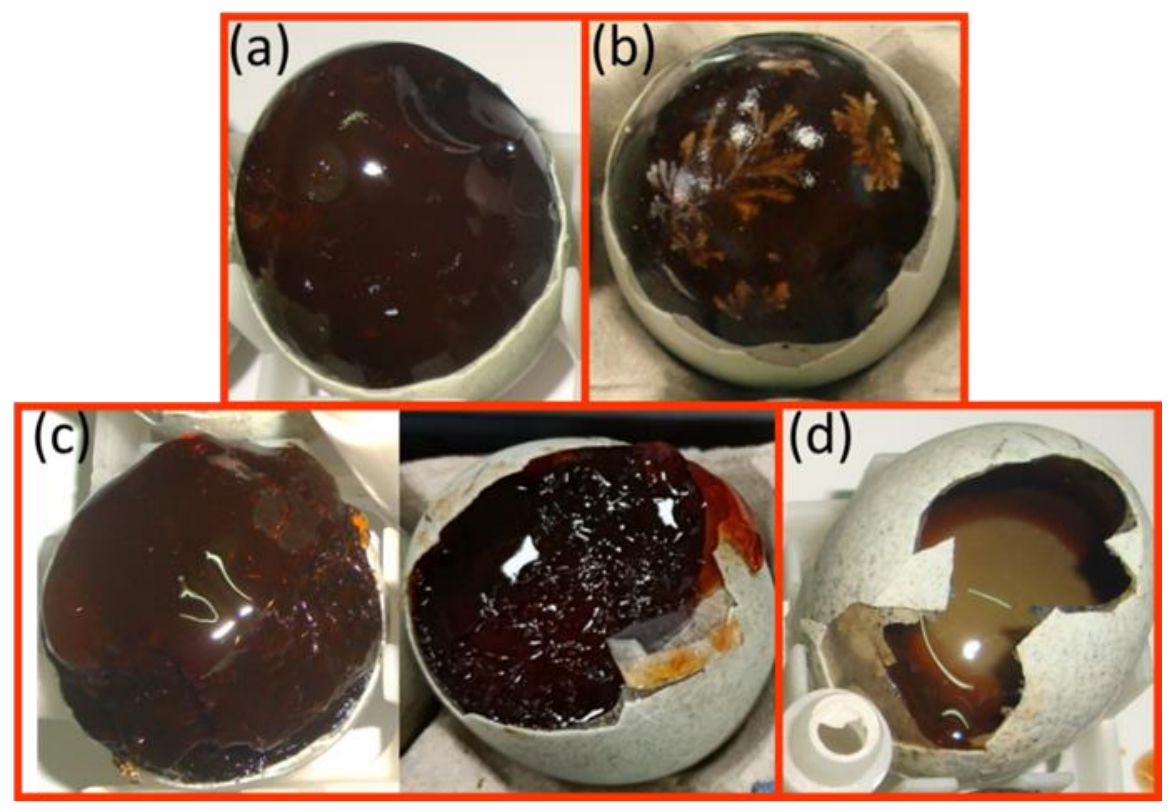

Figure 1. Preserved eggs after the removal of the shell: (a) a gelled egg and (b) the small end of a gelled egg showing the 'pine flower' pattern in the albumen; $(\mathbf{c}, \mathbf{d})$ viscid and liquid non-gelled eggs, respectively.

(a)
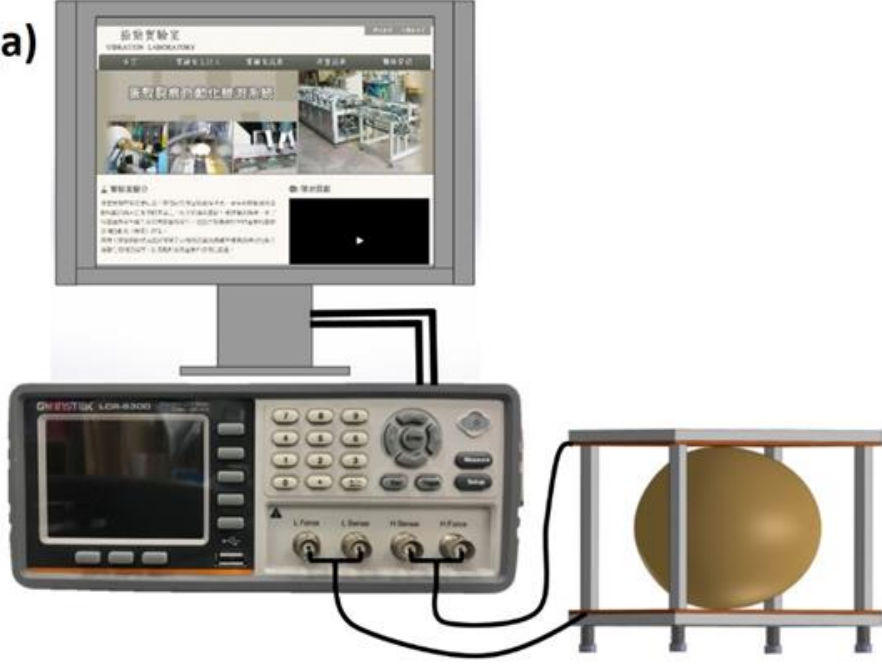

(b)

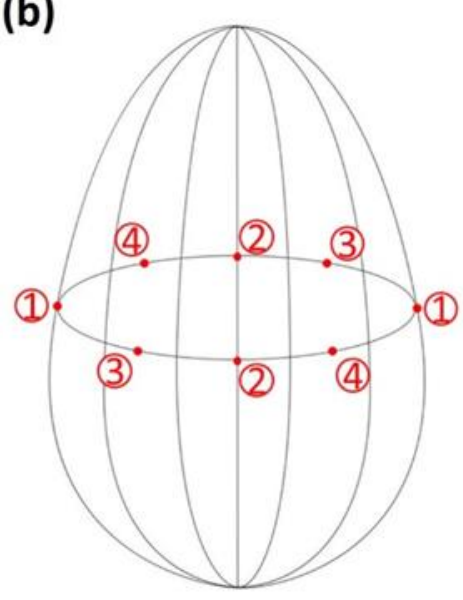

Figure 2. (a) Schematic of the measurement setup; (b) positions where the parallel plate capacitor was placed during the measurements. 


\subsection{Dielectric Analysis}

The dielectric substance generates an external electric field, the internal charges in the material move in a given direction under the action of the electric field, and the charge is redistributed over the surface of the dielectric material. Then, electrons inside the dielectric cannot move freely, which means that the dielectric substance does not conduct electricity under normal circumstances; however, a dielectric material is not necessarily an insulator. When the gap between two conducting electrodes is filled by a dielectric, more energy is stored than would be stored in the absence of the dielectric. The dielectric constant can be used to express the ability of a material to store energy. The dielectric constant is not a fixed value; it depends on factors such as the frequency, temperature, and molecular structure of the material.

The change in the capacitance of a capacitor mainly depends on the dielectric constant, $\varepsilon^{\prime}$, of the material between the two electrode plates, as well as the geometry, size, and relative positions of the two electrodes. Equation (1) is the basic capacitor equation:

$$
\mathrm{C}=\frac{\varepsilon^{\prime} \varepsilon_{0} S}{d}
$$

where $C$ is the capacitance (units: Farad (F)), $\varepsilon^{\prime}$ is the relative permittivity, $\varepsilon_{0}$ is the vacuum permittivity $\left(8.85 \times 10^{-12} \frac{\mathrm{F}}{\mathrm{m}}\right), S$ is the plate area $\left(\mathrm{m}^{2}\right)$, and $\mathrm{d}$ is the distance between the two plates $(\mathrm{m})$.

The dielectric properties will change as the frequency of the applied alternating voltage changes. Because of the interaction between the external electric field and the sample, the dielectric constant will also change. The electrical impedance $(Z)$ under the alternating voltage is composed of a real part-the resistance, $R$-and an imaginary part-the reactance, $X$-and can be expressed by Equation (2). The relationship between the alternating voltage and the current depends on the impedance and phase angle of the impedance, $\theta$; the resistance and reactance are given by $Z \cos \theta$ and $Z \sin \theta$, respectively [15]:

$$
Z=R+j X
$$

and

$$
Z=Z e^{j \theta}=Z(\cos \theta+j \sin \theta)
$$

The loss angle, $\delta$, is the same as the angle $(\theta)$ between the voltage and the current, but with a phase difference of $90^{\circ}$. The loss factor can also be expressed in terms of $\tan \delta$ (Equation (5)):

$$
\delta=\frac{\pi}{2}-\theta
$$

and

$$
\text { Loss factor }(D)=\frac{R}{X}=\tan \delta
$$

\subsection{RLC Resonance Circuit Analysis}

Resistance, inductance, and capacitance can be connected in series to form a circuit. An RLC circuit such as this produces damped oscillations, and it can be represented by the RLC resonance circuit equation:

$$
\mathrm{L} \frac{d^{2} i}{d t^{2}}+\mathrm{R} \frac{d i}{d t}+\frac{1}{C} i=0
$$

the solution to which can be written as

$$
i(t)=A_{1} e^{-\omega_{0}\left(\zeta+\sqrt{\zeta^{2}-1}\right) t}+A_{2} e^{-\omega_{0}\left(\zeta-\sqrt{\zeta^{2}-1}\right) t}
$$


When the damping factor $\zeta<1$, the system is termed an under-damped system, which can also be represented by

$$
i(t)=C e^{-\alpha t} \cos \left(\omega_{d} t+\theta\right)
$$

where $\alpha$ is the RLC series circuit. The rate of attenuation of the oscillations in the current during the transient response can be written as

$$
\alpha=\frac{R}{2 L}
$$

\subsection{Statistical Analysis}

A BN classifier is a probabilistic graphical model. A directed acyclic graph is composed of a directed arc that represents the probability condition relationship between the node representing the variable and the target node. The nodes in the structure represent a set of random variables, $X=X 1, \ldots, X n$, and the directed edges connecting the two nodes represent the dependencies between the variables [16]. Given the graph structure and variable data set, a conditional probability table can be established, which shows the degree of dependence between the variables. In this study, the BN classifier in the WEKA 3.8 software was used to classify the gelling quality of the preserved eggs based on the impedance, resistance, reactance, and decay rate of an RLC circuit. The BN search employed the $\mathrm{k} 2$ algorithm.

The confusion matrix and receiver operating characteristic (ROC) curve are indicators of the effectiveness of classification results. These methods are often used in clinical medical diagnosis and binary data judgment related to the diagnosis of diseases $[17,18]$. In this study, the preserved eggs were divided into two categories: completely gelled and incompletely gelled. The shells of the preserved eggs were removed after the measurements were completed. Then, the results were compared with the real gelling state for the classification. In the case of completely gelled eggs, if the results of the measurements agreed with the real status of an egg, then the result was classified as TP (true positive). For incompletely-gelled eggs, if the measurements also determined that an egg was incompletely gelled, the result was classified as TN (true negative). If the results of the measurements showed that an egg was completely gelled, but in reality, the egg was not completely gelled, the result was classified as FP (false positive). Finally, if an egg was completely gelled, but the results of the measurements showed that it was incompletely gelled, the result was classified as FN (false negative). These four possibilities can be expressed as a $4 \times 4$ confusion matrix [19] (Metz, 1978), as given in Table 1. Using this table, the accuracy (10) and precision (11) of the dielectric measurement technique could be determined.

$$
\begin{gathered}
\text { Accuracy }=\frac{\mathrm{TP}+\mathrm{TN}}{\mathrm{TP}+\mathrm{TN}+\mathrm{FP}+\mathrm{FN}} \\
\text { Precision }=\frac{\mathrm{TP}}{\mathrm{TP}+\mathrm{FP}} \\
\text { Sensitivity }=\frac{\mathrm{TP}}{\mathrm{TP}+\mathrm{FN}}
\end{gathered}
$$

Table 1. Confusion matrix for the preserved egg classification results.

\begin{tabular}{cccc}
\hline & & \multicolumn{2}{c}{ Predicted State } \\
\cline { 2 - 4 } & State & Gelled & Non-Gelled \\
\hline \multirow{2}{*}{ Actual state } & Gelled & TP & FN \\
& Non-gelled & FP & TN \\
\hline
\end{tabular}

In graphs showing the ROC, the sensitivity is plotted on the $y$-axis and the specificity is plotted on the x-axis. The area under the curve in such a graph is called the area under the curve of the ROC (AUC ROC). The AUC index is used to evaluate the accuracy of the 
classification, and it exhibits a value between 0 and 1 . The larger the value of this index, the more accurate the classification. In the case of random guessing with two choices, the value will not be lower than 0.5; as such, in the case of a real classifier, the AUC will also not be less than 0.5 [20]. Cohen's kappa [21], which is another indicator used for the evaluation of the quality of classifications, is defined according to Equation (13). If the value of this indicator is less than 0.40 , the classification lacks reliability and consistency. Values of $0.41-0.60$ indicate that the classification is moderately reliable and consistent, and values of $0.61-0.80$ indicate reliable classification with excellent consistency. A value greater than 0.80 means that the classification is almost perfect [22]. The definition of Cohen's kappa is

$$
\kappa=\frac{p_{0-} p_{c}}{1-p_{c}}
$$

where $p_{0}=\frac{\mathrm{TP}+\mathrm{TN}}{\mathrm{TP}+\mathrm{FP}+\mathrm{FN}+\mathrm{TN}}$ and $p_{c}=\frac{(\mathrm{TP}+\mathrm{FP})(\mathrm{TP}+\mathrm{FN})+(\mathrm{FN}+\mathrm{TN})(\mathrm{FP}+\mathrm{TN})}{(\mathrm{TP}+\mathrm{FP}+\mathrm{FN}+\mathrm{TN})^{2}}$.

\section{Results and Discussion}

\subsection{Impedance Spectroscopy Analysis}

In this study, an LCR meter and parallel plate capacitors were used to measure the impedance spectra of completely-gelled and incompletely-gelled preserved eggs between 2 and $300 \mathrm{kHz}$. Figure 3 shows that the impedance of gelled and non-gelled preserved eggs decreases with an increase in the frequency. Moreover, at the same frequency, eggs that are not completely gelled exhibit lower impedance than completely-gelled eggs. The impedance is composed of real resistance and imaginary reactance. In this section, the resistance and reactance of completely- and incompletely-gelled preserved eggs will be analyzed.

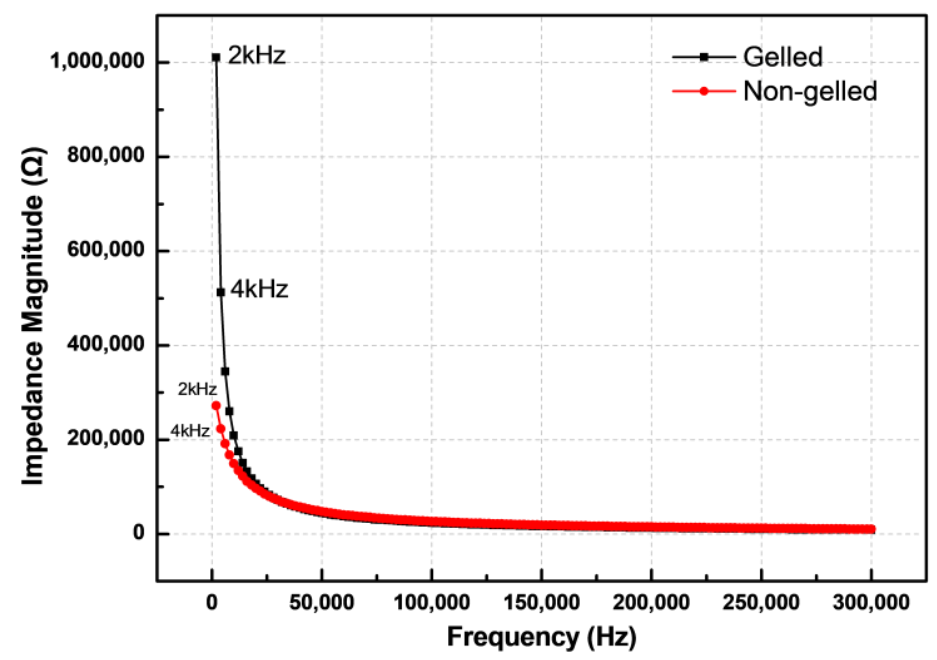

Figure 3. Plots of impedance against frequency for completely-gelled and incompletely-gelled preserved eggs.

Figure $4 \mathrm{~b}$ reveals significant differences between the Nyquist plots of completely- and incompletely-gelled preserved eggs. Figure $4 \mathrm{a}$ shows that the reactance of gelled-preserved eggs and incompletely-gelled preserved eggs are both negative in the range of $2-300 \mathrm{kHz}$, which indicates that both gelled-and incompletely-gelled preserved eggs exhibit capacitive characteristics. In Figure 4, the Nyquist plot of the reactance versus the resistance of completely-gelled preserved eggs in the range of $2-300 \mathrm{kHz}$ gives an oblique line. The inside of a preserved egg that is completely gelled is solid, and the charged ions inside the preserved egg will separate under the action of an electric field. However, because the inside of the egg is solid, the charged ions cannot move to the positive or negative pole, so the Nyquist plot of the reactance and resistance produces an oblique straight line. This process represents diffusion effects. The Nyquist plot of incompletely-gelled preserved eggs 
is a semicircle, as shown in Figure 4. Under the action of an electric field with a frequency between 2 and $300 \mathrm{kHz}$, the internal ions of incompletely-gelled preserved eggs will quickly separate. Because the ions inside the liquid interior of incompletely-gelled preserved eggs can easily move to the electrodes at both ends, the charge transfer process will appear as a semicircular arc in the Nyquist plot. The preserved eggs' measurement frequency range of 2-300 kHz corresponds to beta dispersion. The beta dispersion frequency associated with the polarization of cellular membranes, protein, and other organic macromolecules ranges from $1 \mathrm{kHz}$ to several $\mathrm{MHz}$ [23]. The impedance of gelled and non-gelled preserved eggs will change, as shown in Figure 4. The inference is that the degree of albumen gelation in preserved eggs is different.

(a)

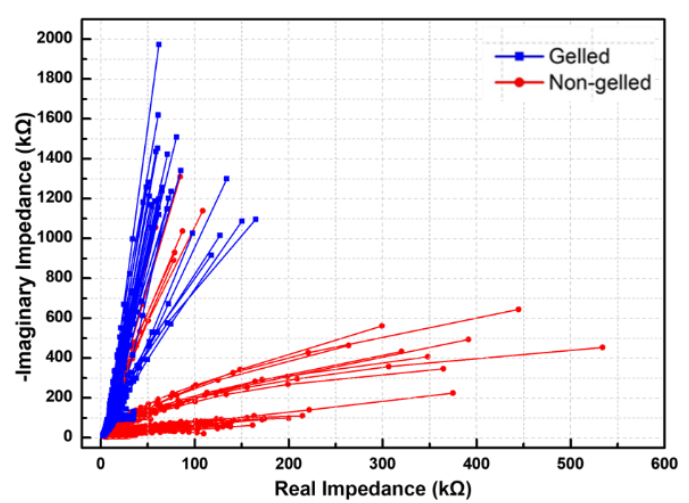

(c)

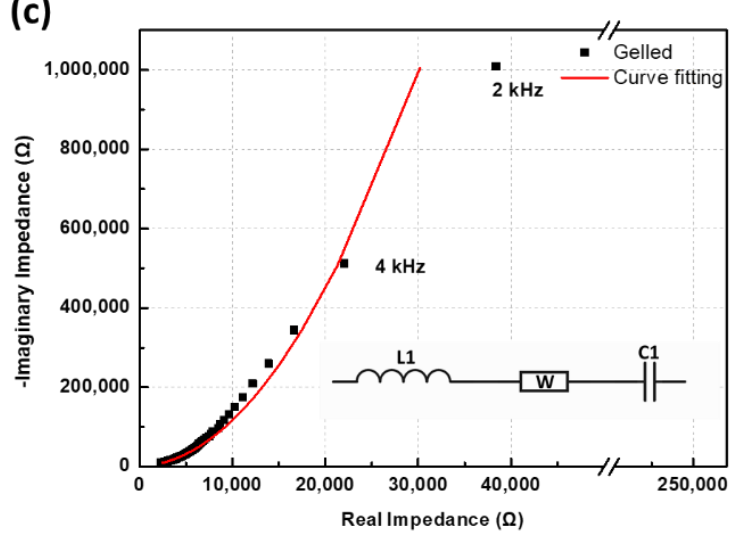

(b)

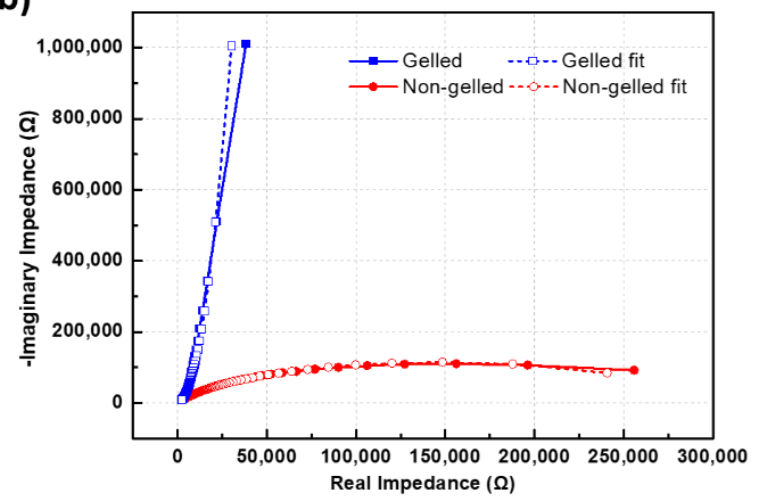

(d)

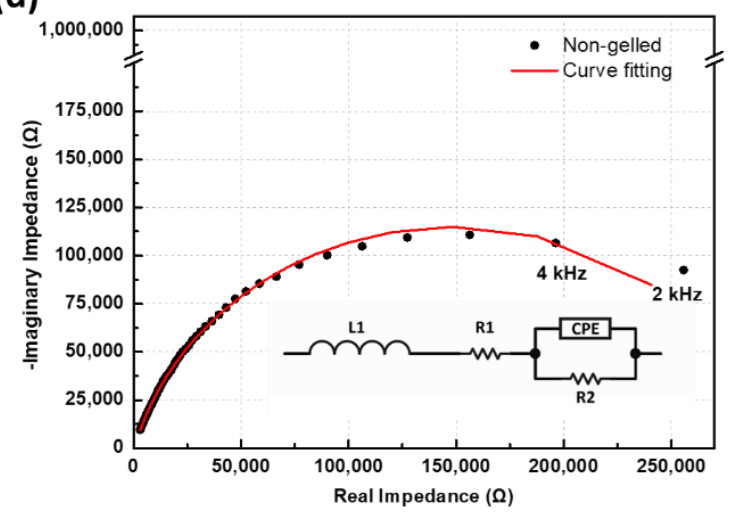

Figure 4. (a) Nyquist plots (impedance curves) for 30 completely- and 30 incompletely-gelled preserved eggs. (b) Nyquist plots from Figure 4. (a) Two fitted impedance curves for completely- and incompletely-gelled preserved eggs. Also shown are the fitted impedance curves (range: $2-300 \mathrm{kHz}$ ) obtained by simulating the equivalent circuit of (c) completely-gelled and (d) incompletely-gelled preserved eggs.

The capacitive characteristics of real electronic components can be used to analyze completely- and incompletely-gelled preserved eggs. At low frequencies, the impedance and reactance of incompletely-gelled preserved eggs will be lower than those of completelygelled preserved eggs. By contrast, the resistance of incompletely-gelled preserved eggs is greater than that of completely-gelled preserved eggs. Normal capacitors tend to block direct current while allowing alternating current to pass. In the case of a low-frequency alternating current, the resistance of a capacitor will be relatively large, thus preventing direct current from passing, whereas the reactance will be small, allowing alternating current to pass.

In terms of electronic characteristics, an incompletely-gelled preserved egg is similar to a capacitor. Compared with a real capacitor, the resistance of resistor R2, which is shown connected in parallel to the constant phase element (CPE) in Figure 4d, will be very large. $\mathrm{R} 2$ can be regarded as a bleeder resistor, and it quickly consumes the electrical energy 
in the preserved egg, which means that it does not exhibit the ability to store energy in the same way as an electronic capacitor. Compared with incompletely-gelled preserved eggs, the electrical resistance of completely-gelled preserved eggs is minimal, and the reactance is large. In the case of real electronic capacitors, when the electrolyte inside the capacitor is lost or has dried up, the capacitor will form a short circuit. Then, the internal resistance will be very small, and the reactance will be large; this will cause the capacitor to heat up and suffer damage when an alternating-current voltage passes through it. In a completely-gelled preserved egg, no watery albumen is present, which means that the resistance is minimal under a low-frequency alternating-current voltage; therefore, the reactance is very large, which is similar to the case of a capacitor with no electrolyte inside.

The gelling of preserved eggs is a process of continuous and gradual change, and the reactance and resistance of preserved eggs changes during this process. In the present study, the researchers speculated that the reactance of incompletely-gelled preserved eggs would be lower than that of completely-gelled preserved eggs. The reason for this is that the inside of incompletely-gelled preserved eggs consists of liquid albumen; therefore, the ions inside the egg can move easily under the influence of alternating current, giving a small reactance value. After the same marinating time, both completely-gelled and incompletely-gelled preserved eggs can be produced. This is because the gelling may fail, owing to the excessive alkalinity of the pickling liquid or the excessive absorption of the liquid by the egg during the pickling process. The albumen inside preserved eggs that are excessively alkalized will become liquid, and the reactance will be low.

According to the National Standard of the Republic of China, CNS 2174 (Taiwan, 1986), regarding testing methods for alkalized eggs, the albumen at the small end of preserved eggs should have liquefied and be sticky. The yolk should solidify, and if the conditions during the pickling process are excessively alkaline, the gelling process can fail or be incomplete. The characteristics of an incompletely-gelled preserved egg include the albumen failing to gel, and a solidified egg yolk. According to the CNS 2174 guidelines, such an egg will be considered over-alkalized, or the consideration will be that the pickling liquid was too alkaline. The Nyquist plots for completely- and incompletelygelled preserved eggs shown in Figure $4 c, d$, respectively, were analyzed and simulated using noncommercial impedance spectroscopy software in order to establish the equivalent circuits for the two different states of preserved eggs. These equivalent circuits were series circuits comprising resistance, inductance, and capacitance. The circuits were also similar to the equivalent circuits for electronic capacitors.

Element $W$ in Figure 4c is known as the Warburg element, which is composed of the diffusion admittance $\left(Y_{0}\right)$ and the diffusion time constant $(B)$. These components are related to changes in the Warburg impedance, as given by Equation (14):

$$
Z_{w}=\frac{1}{Y_{0}} \tanh (B j \omega)
$$

where $\omega$ is the angular frequency. When the admittance value, $Y_{0}$, increases, the total impedance decreases and the diffusion time constant, $B$, decreases. This may be due to the higher concentration of charged ions in the medium, which increases the total conductivity, affects $Y_{0}$, and reduces the value of $B$.

The admittance of the CPE shown in Figure 4d can be calculated using Equation (15):

$$
Y_{C P E}=\frac{1}{Z_{C P E}}=Q_{0}(j \omega)^{n}
$$

Figure 4c shows the equivalent circuit element after the curve fitting, in which $\mathrm{L} 1=218 \mathrm{pH}$. The Warburg impedance elements used were $4.786 \frac{\mathrm{M} \Omega}{\sqrt{s}} ; \mathrm{C} 1$ is a capacitor with a capacitance of $81.67 \mathrm{pF}$. Figure $4 \mathrm{~d}$ shows the values of the components of the equivalent circuit after the curve fitting. L1 is an inductor with an inductance of $583.7 \mu \mathrm{H}$, resistor R1 exhibits a resistance of $46.25 \Omega$, resistor R2 exhibits a resistance of $298.3 \mathrm{k} \Omega$, and 
the value of $Q_{0}$ for the CPE is $503.5 \mathrm{ps}^{n} \cdot \Omega^{-1}$, where $n$ is 0.8361 . When $n=1$, the CPE is an ideal capacitor; when $n=-1$, it is an inductor; and when $n=0$, it is a pure resistor. When $n>0.8$, the CPE demonstrates the characteristics of a capacitor.

To summarise, we can regard a preserved egg as a capacitor. A fully-gelled preserved egg has small resistance and large reactance, similar to a bad capacitor in which the electrolyte is dried up, as shown in Figure 4c. An incompletely-gelled preserved egg has a large resistance and small reactance, similar to a normal capacitor with the presence of an electrolyte inside, as shown in Figure 4d. Therefore, the impedance measurement was conducted under the AC signal at the same frequency, and the impedance of a fully-gelled preserved egg was greater than an incompletely-gelled preserved egg, as shown in Figure 3.

\subsection{Analysis of the Decay of the Circuit Transient Response}

Chen et al. (2011) measured the rate of decay of the oscillations of a preserved egg by tapping a preserved egg and judging the quality of the preserved egg's gel from the decay rate [12]. The rate of decay of the oscillations for a completely-gelled egg was smaller than that for an incompletely-gelled preserved egg.

In Section 3.1 of this paper, the researchers described how the impedance of preserved eggs was used to simulate the equivalent circuits for completely- and incompletely-gelled preserved eggs, which are shown in Figure $4 \mathrm{c}, \mathrm{d}$, respectively. The eggs here are equivalent to a series combination of a resistor, capacitor, and inductor, which can be expressed as Equation (6). The equation for an RLC resonance circuit (Equation (6)) is similar to the equation for a damped mechanical oscillation. The transient response exhibits an oscillation-signal decay rate of $\alpha$, which can be calculated using Equation (9).

In order to obtain the resistance, capacitance, and inductance values of the preserved egg, the resistance, inductance, and capacitance of the RLC resonance circuit were measured using an LCR meter. The measured inductance value of the LCR meter was negative. The main reason for this was that the reactance was negative. This can be deduced from Equation (16) which gives the inductance:

$$
\mathrm{L}=\mathrm{X} / 2 \pi f
$$

where $X=X_{L}-X_{c}, L$ is the inductance, $X$ is the total reactance, $f$ is the frequency, $X_{L}$ is the inductive reactance, and $X_{c}$ is the capacitive reactance.

The reactance measured using the LCR meter was the total reactance. The capacitive reactance of a preserved egg is greater than its inductive reactance, which means that the total reactance is negative. The inductance is also negative, and a preserved egg exhibits relatively few inductive components. As the inductance value becomes very small at a frequency of $6 \mathrm{kHz}$, the inductance, capacitance, and resistance measured at a frequency of $6 \mathrm{kHz}$ were used in the equation for the RLC resonance circuit. Figure 5 shows the rate of energy attenuation for the transient response of the RLC circuit at a frequency of $6 \mathrm{kHz}$; Figure $5 \mathrm{a}, \mathrm{b}$ correspond to completely- and incompletely-gelled eggs, respectively. It can be seen that the decay rate for completely-gelled eggs is less than $\sim 2.3 \mathrm{~s}^{-1}$, whereas the average decay rate of incompletely-gelled preserved eggs is more than $\sim 8 \mathrm{~s}^{-1}$. Thus, a significant difference exists between these two rates.

The amount of energy stored in completely-gelled preserved eggs decays slowly, whereas the energy stored in incompletely gelled eggs decays quickly. It can be seen from Figure 4c,d that the electrical resistance of completely-gelled preserved eggs is small, whereas that of incompletely-gelled preserved eggs is large. When the transient current passes through a completely-gelled preserved egg, the resistance is small. The transient current is not easily blocked and consumed, so it decays slowly.

The resistance of incompletely-gelled preserved eggs is large, which means that the current can easily be blocked and consumed; thus, the current decays fast. The RLC resonance circuit equation obtained using the dielectric method to measure the decay rate of preserved eggs, and the damping oscillation equation for the measurement of the oscillations produced by tapping preserved eggs [12] exhibit the same decay rate. The decay 
rate for completely-gelled preserved eggs is low, whereas the decay rate for incompletely gel-preserved eggs is high.
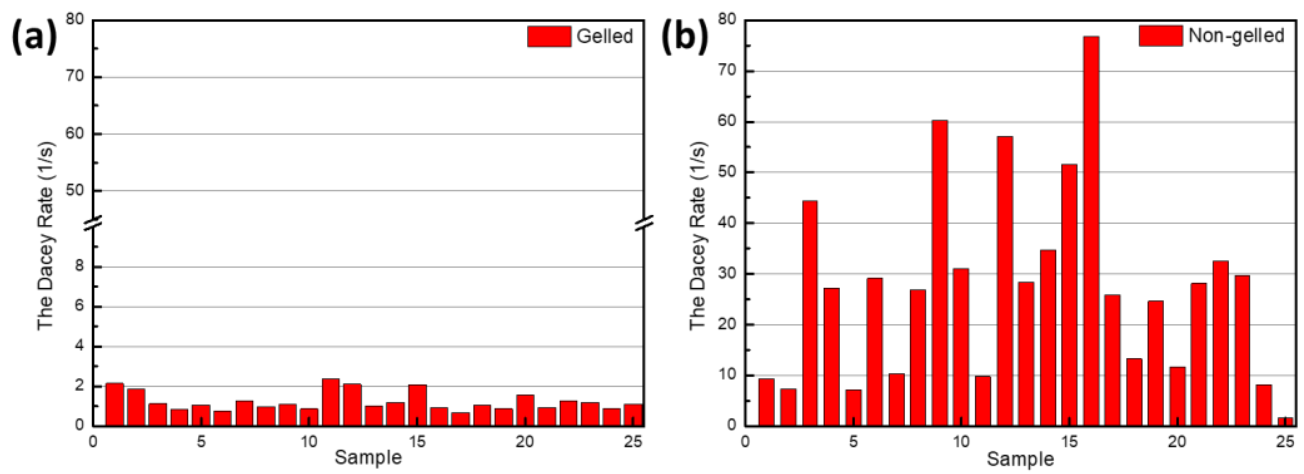

Figure 5. Rates of current decay at $6 \mathrm{kHz}$ for (a) completely-gelled and (b) incompletely-gelled preserved eggs.

\subsection{Classification of Preserved Egg Gel Based on Impedance}

Impedance comprises resistance, which is real, and reactance, which is imaginary. From the Nyquist plot shown in Figure $4 d$, it can be seen that, for incompletely-gelled eggs, the maximum value of the imaginary reactance in the range $2-300 \mathrm{kHz}$ occurs between approximately 6 and $8 \mathrm{kHz}$. Therefore, in this study, the impedance, resistance, and reactance at $6 \mathrm{kHz}$ were selected as the characteristic variables for the classification of preserved eggs. A BN classifier was used to classify the quality of the gel in the preserved eggs. The 60 preserved eggs were divided into two types-completely gelled and incompletely gelled-and the gel state of the preserved eggs was classified, as shown in Figure 1. The preserved egg used the impedance, resistance, and reactance as the characteristic classification variables, as given in Tables 2 and 3. The training results are given in Table 2. The training accuracy obtained using the BN classifier using impedance as the characteristic variable was $95 \%$. The training accuracy obtained using the resistance and reactance as characteristic variables was $96.7 \%$.

Table 2. Training results obtained for the BN classifier using the impedance, resistance, and reactance at $6 \mathrm{kHz}$, and the decay rate at $6 \mathrm{kHz}$ as the characteristic variables.

\begin{tabular}{ccccc}
\hline Variables & Kappa Statistic & $\begin{array}{c}\text { Training } \\
\text { Accuracy (\%) }\end{array}$ & Precision (\%) & Sensitivity (\%) \\
\hline Impedance (6 kHz) & 0.90 & 95.0 & 96.6 & 93.3 \\
Resistance (6 kHz), & 0.93 & 96.7 & 96.7 & 96.7 \\
Reactance (6 kHz) & 0.90 & 95.0 & 91.0 & 100 \\
Decay rate (6 kHz) & & & \\
\hline
\end{tabular}

Table 3. Test results for the BN classifier using the impedance, resistance, and reactance at $6 \mathrm{kHz}$, and the decay rate at $6 \mathrm{kHz}$ obtained using a trained BN classifier.

\begin{tabular}{|c|c|c|c|c|c|}
\hline Variables & $\begin{array}{c}\text { Kappa } \\
\text { Statistic }\end{array}$ & $\begin{array}{c}\text { Testing } \\
\text { Accuracy (\%) }\end{array}$ & $\begin{array}{l}\text { Precision } \\
(\%)\end{array}$ & $\begin{array}{c}\text { Sensitivity } \\
(\%)\end{array}$ & $\begin{array}{l}\text { AUC } \\
\text { ROC }\end{array}$ \\
\hline Impedance (6 kHz) & 0.40 & 70.7 & 80.0 & 51.0 & 0.70 \\
\hline $\begin{array}{l}\text { Resistance }(6 \mathrm{kHz}) \text {, } \\
\text { Reactance }(6 \mathrm{kHz})\end{array}$ & 0.62 & 81.0 & 81.1 & 78.2 & 0.88 \\
\hline Decay rate $(6 \mathrm{kHz})$ & 0.64 & 81.9 & 75.8 & 90.9 & 0.82 \\
\hline
\end{tabular}

Another batch of 116 preserved eggs was used to test the trained BN classification model. The classification accuracy obtained using this batch of eggs was $70.7 \%$ for the BN model, which was trained using impedance as the characteristic variable. The classification 
model trained with resistance and reactance as the characteristic variables achieved a classification accuracy of $81.0 \%$. When the decay rate at $6 \mathrm{kHz}$ was used as the characteristic variable, as given in Table 3, the classification accuracy was $81.9 \%$. Therefore, if the impedance alone is used for classification and prediction, a large number of misjudgments will occur, and some completely-gelled eggs will be classified as incompletely-gelled eggs. The classification results are given in the confusion matrix in Table 4. Overall, the use of the $\mathrm{BN}$ classifier with resistance and reactance as the characteristic variables produced the best classification accuracy.

Table 4. Confusion matrix for the real classification performed after training the BN classifier.

\begin{tabular}{|c|c|c|c|c|}
\hline \multirow{2}{*}{ Variables } & & & \multicolumn{2}{|c|}{ Predicted State } \\
\hline & & & Gelled & Non-Gelled \\
\hline \multirow{2}{*}{ Impedance $(6 \mathrm{kHz})$} & \multirow{6}{*}{ Actual state } & Gelled & 28 & 27 \\
\hline & & Non-gelled & 7 & 54 \\
\hline Resistance (6 kHz), & & Gelled & 43 & 12 \\
\hline Reactance $(6 \mathrm{kHz})$ & & Non-gelled & 10 & 51 \\
\hline \multirow{2}{*}{ Decay rate $(6 \mathrm{kHz})$} & & Gelled & 50 & 5 \\
\hline & & Non-gelled & 16 & 45 \\
\hline
\end{tabular}

The oscillations of the gelled preserved egg can be felt by the palm using the traditional finger tapping. At the same time, this approach does not work for non-gelled preserved eggs. At an actual factory, workers may tap several thousand preserved eggs per day with their fingers. As the working time increases, the sensitivity of the manual tapping detection will decrease. During the testing process, personal experience and sensations will affect the determination of the gelling and non-gelling of preserved eggs, and there will be differences. The proposed method allows the exclusion of human factors, and a relatively higher detection accuracy compared with the traditional tapping method. The proposed method is different from that of Chen et al. (2011). More specifically, Chen et al. (2011) proposed the use of a plastic stick-tapping method to determine the quality of the gelling in preserved eggs. In their method, the shell was not removed, and the accelerometer was used to measure the oscillations in the eggs [12]. The authors demonstrated that the detection accuracy of gelled and non-gelled preserved eggs could reach $96.7 \%$. The dielectric impedance method employed parallel plate capacitors for the detection. In particular, the parallel plate electrodes touched the equator of the preserved egg. Thus, the signal may be affected by the egg yolk and air factors. It was mainly measured around the equator's gel condition; moreover, it was difficult to detect the gel condition of the small end of the preserved egg in detail. Therefore, misjudgment occurred, and the detection accuracy was lower than that of Chen et al. [12].

\section{Conclusions}

In this study, preserved eggs were classified as being either completely- or incompletelygelled based on their real resistance and imaginary reactance, which are the two components of impedance, and using machine learning. This method-based on dielectric impedance measurements - is simple, cheap, and accurate. The measured resistance and reactance within the frequency range of $2-300 \mathrm{kHz}$ were plotted on a Nyquist diagram, and curve fitting was performed in order to obtain the equivalent circuits for completelyand incompletely-gelled preserved eggs. The results of the decay rate obtained by tapping oscillation or using an RLC resonance circuit are similar. The decay rate for completelygelled eggs was low, whereas the decay rate for incompletely-gelled eggs was high. Using both resistance and reactance as the variables, a BN achieved a classification accuracy of $81.0 \%$, which was greater than that obtained using only impedance used as the variable. The nondestructive dielectric technique used in this research provides an objective and simple way of determining the quality of preserved egg gel, and it proves that dielectric impedance can be used to classify preserved egg gel. In the future, the use of dielectric 
measurement techniques in automatic systems for the inspection of preserved eggs will be possible.

Author Contributions: C.-H.L., C.-C.H. and C.-W.C. conceived and designed the experiments; C.-H.L. and C.-C.H. performed the experiments; C.-H.L., C.-H.H., C.-C.H. and C.-W.C. analyzed the data; C.-H.L., C.-H.H. and C.-W.C. wrote the paper; C.-H.L., C.-H.H., C.-C.H. and C.-W.C. reviewed and edited the paper. All authors have read and agreed to the published version of the manuscript.

Funding: The authors would like to thank the Ministry of Science and Technology, Taiwan. (Grant Nos. MOST 109-2321-B-025-001).

Acknowledgments: The authors thank the Ministry of Science and Technology of the Republic of China for the financial support.

Conflicts of Interest: The authors declare no conflict of interest.

\section{References}

1. Chang, H.M.; Tsai, C.F.; Li, C.F. Changes of amino acid composition and lysinoalanine formation in alkali-pickled duck eggs. J. Agric. Food Chem. 1999, 47, 1495-1500. [CrossRef]

2. Ye, C.M. Technique of preserved egg processing and edible security. Agric. Sci. Technol. Equip. 2015, 1, 62-63. [CrossRef]

3. Zhang, X.W.; Jiang, A.M.; Chen, M.T.; Ockerman, H.W.; Chen, J.J. Effect of different alkali treatments on the chemical composition, physical properties, and microstructure of pidan white. J. Food Sci. Technol. Mysore 2015, 52, 2264-2271. [CrossRef]

4. Zhao, Y.; Chen, Z.Y.; Li, J.K.; Xu, M.S.; Shao, Y.Y.; Tu, Y.G. Changes of microstructure characteristics and intermolecular interactions of preserved egg white gel during pickling. Food Chem. 2016, 203, 323-330. [CrossRef] [PubMed]

5. Watanabe, T.; Ando, Y.; Orikasa, T.; Kasai, S.; Shiina, T. Electrical impedance estimation for apple fruit tissues during storage using Cole-Cole plots. J. Food Eng. 2018, 221, 29-34. [CrossRef]

6. Yang, N.; Jin, Y.M.; Wang, H.Y.; Duan, X.; Xu, B.C.; Lin, Z.Y.; Xu, X.M. Evaluation of conductivity and moisture content of eggs during storage by using transformer method. J. Food Eng. 2015, 155, 45-52. [CrossRef]

7. Ghaderi, M.; Banakar, A.; Masoudi, A.A. Using dielectric properties and intelligent methods in separating of hatching eggs during incubation. Measurement 2018, 114, 191-194. [CrossRef]

8. Soltani, M.; Omid, M. Detection of poultry egg freshness by dielectric spectroscopy and machine learning techniques. LWT Food Sci. Technol. 2015, 62, 1034-1042. [CrossRef]

9. Ibba, P.; Falco, A.; Abera, B.D.; Cantarella, G.; Petti, L.; Lugli, P. Bio-impedance and circuit parameters: An analysis for tracking fruit ripening. Postharvest Biol. Technol. 2020, 159. [CrossRef]

10. Castro-Giraldez, M.; Fito, P.J.; Chenoll, C.; Fito, P. Development of a dielectric spectroscopy technique for the determination of apple (Granny Smith) maturity. Innov. Food Sci. Emerg. Technol. 2010, 11, 749-754. [CrossRef]

11. Polak, T.; Polak, A.; Poklar, N.U.; Segatin, N. Electrical admittance and dielectric properties of whipping cream. J. Food Eng. 2020, 278. [CrossRef]

12. Chen, Y.C.; Hu, M.L.; Cheng, C.W. Applying non-destructive techniques to inspect preserved egg products by decay rates. J. Food Eng. 2011, 104, 30-35. [CrossRef]

13. Weng, Y.-K.; Chen, J.; Cheng, C.-W.; Chen, C. Use of Modern Regression Analysis in the Dielectric Properties of Foods. Foods 2020, 9, 1472. [CrossRef]

14. Figueiredo Neto, A.; Cárdenas Olivier, N.; Rabelo Cordeiro, E.; Pequeno de Oliveira, H. Determination of mango ripening degree by electrical impedance spectroscopy. Comput. Electron. Agric. 2017, 143, 222-226. [CrossRef]

15. Ando, Y.; Mizutani, K.; Wakatsuki, N. Electrical impedance analysis of potato tissues during drying. J. Food Eng. 2014, 121 , 24-31. [CrossRef]

16. Atoui, M.A.; Cohen, A.; Verron, S.; Kobi, A. A single Bayesian network classifier for monitoring with unknown classes. Eng. Appl. Artif. Intell. 2019, 85, 681-690. [CrossRef]

17. Ma, H.; Bandos, A.I.; Gur, D. On the use of partial area under the ROC curve for comparison of two diagnostic tests. Biom. J. 2015, 57, 304-320. [CrossRef]

18. Zweig, M.H.; Campbell, G. Receiver-operating characteristic (roc) plots-a fundamental evaluation tool in clinical medicine. Clin. Chem. 1993, 39, 561-577. [CrossRef] [PubMed]

19. Metz, C.E. Basic principles of roc analysis. Semin. Nucl. Med. 1978, 8, 283-298. [CrossRef]

20. Fawcett, T. An introduction to ROC analysis. Pattern Recognit. Lett. 2006, 27, 861-874. [CrossRef]

21. Cohen, J. A Coefficient of Agreement for Nominal Scales. Educ. Psychol. Meas. 1960, 20, 37-46. [CrossRef]

22. Landis, J.R.; Koch, G.G. The Measurement of Observer Agreement for Categorical Data. Biometrics 1977, 33, 159-174. [CrossRef] [PubMed]

23. Dean, D.A.; Ramanathan, T.; Machado, D.; Sundararajan, R. Electrical Impedance Spectroscopy Study of Biological Tissues. J. Electrostat. 2008, 66, 165-177. [CrossRef] [PubMed] 